\title{
Application de la résonance magnétique nucléaire aux écoulements diphasiques
}

\author{
par Sylvain Javelot, Jacques Leblond et Christian Baradel \\ du Laboratoire de Physique et Mécanique des Milieux Hétérogènes, Paris
}

\section{INTRODUCTION}

On sait depuis longtemps que la RMN peut être utilisée pour mesurer des déplacements moléculaires. Dès 1965, Stejskal [1] a présenté une analyse théorique assez complète sur les effets de la diffusion moléculaire anisotrope et sur ceux d'un écoulement. Il montrait de quelle manière étaient modifiées l'amplitude et la phase d'un écho de spin en présence de gradients de champ magnétique. Sur cette base, de nombreux travaux expérimentaux ont suivi. La RMN a surtout été appliquée à l'étude de la diffusion moléculaire dans les systèmes complexes [2-4] ; de nombreux exemples d'utilisation de la RMN dans ce domaine ont été présentés par Callaghan [5]. L'analyse des écoulements de fluide grâce à des techniques d'écho de spins a eu un développement plus lent. Les premières études ont essentiellement porté sur des écoulements lents en canal [3, 6-9]. Toutefois, dès 1969, de Gennes [10] établissait que le temps de corrélation des vitesses pouvait être relié à l'atténuation des échos pairs dans une séquence de CarrPurcell [11]. L'analyse par RMN des écoulements turbulents est plus récente. Les travaux publiés sur le sujet [12-14] ont démontré l'avantage d'utiliser la technique d'écho de spins en présence d'un gradient de champ magnétique pour mesurer la distribution de probabilité des vitesses et caractériser les fluctuations des vitesses. Plus récemment, le développement de l'imagerie RMN a impliqué la mise en place de méthodes sophistiquées sans précédent exploitant l'algorithme de Transformation de Fourier Rapide. Cette étape importante a permis d'envisager de nouvelles études sur les écoulements. Il est maintenant possible d'obtenir le profil des vitesses dans une section de canalisation [15]. Plusieurs revues sur l'utilisation de la RMN dans la caractérisation des écoulements ont déjà été publiées [16-20]. Cela dit, très peu de travaux ont été publiés sur l'application de la RMN à la caractérisation des écoulements multiphasiques. La plupart d'entre eux concernent les écoulements multiphasiques en milieux poreux [21-23]. Des écoulements diphasiques en
The results presented in this paper demonstrate the good performance of the PFGSE-NMR to obtain a complete characterization of twophase flows. This method has been applied to air-water flows and also to water-Freon 113 flows where the two liquids are discemible by NMR and can be characterized independently.

canal ont toutefois été rapportés par Lynch \& Segel [24] qui ont mesuré les fractions volumiques des phases dans un écoulement gaz-liquide. Abouelwafa \& Kendall [25] ont développé une technique pour mesurer les vitesses moyennes et les fractions volumiques des phases dans un mélange eau-huile. Krüger et al. [26] ont proposé d'utiliser une mesure du temps d'écoulement des protons dans la bobine de détection de la RMN pour déduire le débit massique dans un écoulement eau-vapeur; leur méthode devrait être applicable sur une gamme très étendue de vitesses et de taux de vide (vitesses de 0,3 à $100 \mathrm{~m} / \mathrm{s}$ ); toutefois elle ne permet pas d'obtenir une information sur le profil des vitesses, ni sur la distribution de probabilité des vitesses dans le canal. Enfin, Majors et al. [27] ont utilisé une technique d'imagerie RMN pour mesurer, en même temps, dans un écoulement lent et stationnaire, les profils de vitesse et de concentration dans l'écoulement d'une suspension de sphères monodisperses de polyéthylène au sein d'une huile visqueuse.

Nous présentons dans cet article, Spinflow, un dispositif RMN qui a été conçu à l'ESPCI pour caractériser les écoulements complexes et en particulier les écoulements multiphasiques. Après une description rapide des principaux éléments du système, nous présenterons la méthode que nous avons utilisée pour accéder aux principales caractéristiques de ces écoulements très particuliers où vitesses et fractions volumiques subissent des fluctuations très importantes. Dans 
un écoulement à une seule phase, la fraction volumique de la phase est évidemment constante et les fluctuations de vitesse sont relativement faibles; elles n'excèdent généralement pas $10 \%$ même dans un écoulement turbulent. Dans un écoulement diphasique eau-air, la fraction volumique du liquide varie très fortement d'un instant à l'autre. Il en est de même des vitesses du liquide; des fluctuations supérieures à $100 \%$ sont couramment observées. On se trouvait devant un problème nouveau pour lequel il a fallu trouver une méthode d'analyse adaptée. Par ailleurs, les fluctuations des fractions volumiques des phases s'accompagnent de fluctuations des propriétés diélectriques du milieu, fluctuations auxquelles sont en général sensibles les anternes (bobines) de détection du signal de RMN. Il a donc fallu concevoir des antennes électromagnétiques, sensibles aux propriétés magnétiques qui nous intéressent, mais insensibles aux propriétés diélectriques des fluides.

Les performances de l'équipement seront présentées sur deux types d'écoulement diphasique en canal, des écoulements «eau-air" et des écoulements «eau-fréon liquide », sur une vaste gamme de fraction volumique $(0-1)$ et jusqu'à des vitesses de $1 \mathrm{~m} / \mathrm{s}$.

\section{DISPOSITIF EXPÉRIMENTAL}

Le dispositif a été construit à partir d'un spectromètre SMIS du type Multispect opérant dans la gamme 2-100 MHz, choisi pour sa flexibilité. Le champ magnétique $B_{0}$ nécessaire à l'analyse de la RMN est obtenu grâce à un électroaimant construit par la société Drusch (voir fig. 1). Il est constitué de deux solénoïdes placés bout à bout et générant dans tout leur volume intérieur un champ de 0,12 Tesla. La fréquence de résonance correspondante pour les protons est de $5 \mathrm{MHz}$. Le solénoïde principal, de

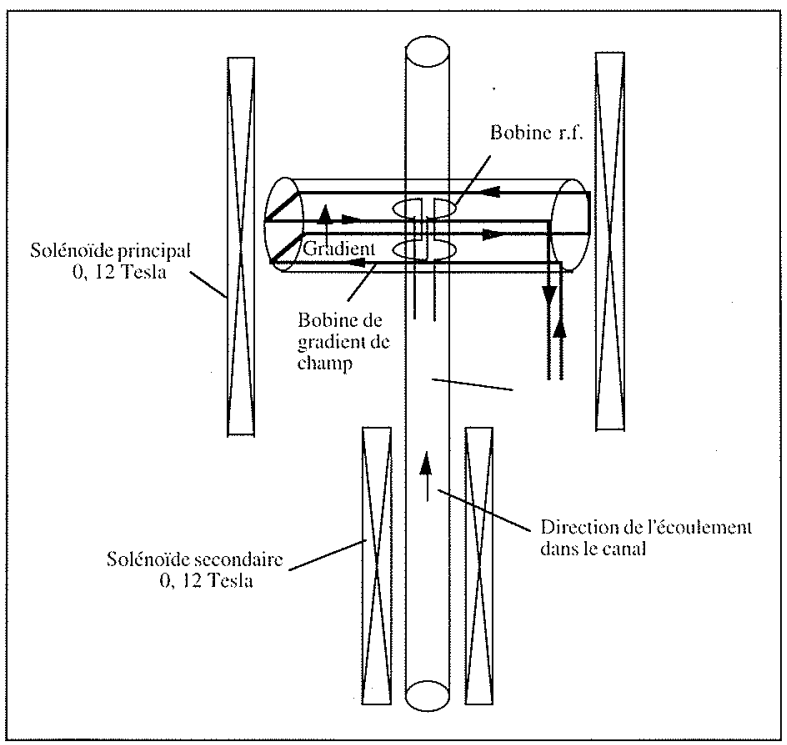

1. Schéma du générateur de champ magnétique comprenant deux solénoïdes plaçés bout à bout. La bobine de gradient de champ magnétique et la bobine r.f. permettent d'appliquer la séquence PFGSE dans la zone de mesure correspondant à un tronçon de canalisation de longueur $L \approx D=50 \mathrm{~mm}$ (diamètre du canal).
$1 \mathrm{~m}$ de haut et de $30 \mathrm{~cm}$ de diamètre intérieur, possède une zone centrale réservée aux mesures de RMN où l'homogénéité est de $\pm 10^{-5} B_{0}$ dans un cylindre de $6 \mathrm{~cm}$ de diamètre et $10 \mathrm{~cm}$ de long. Le solénoïde secondaire de $80 \mathrm{~cm}$ de haut et de $10 \mathrm{~cm}$ de diamètre intérieur, est destiné à la polarisation des spins nucléaires du fluide en écoulement. L'homogénéité n'est que de quelques pour-cent de $B_{0}$. La stabilité temporelle du champ à court terme est de $10^{-6}$ et à long terme de $10^{-5}$.

L'écoulement est étudié dans une canalisation circulaire droite de $50 \mathrm{~mm}$ de diamètre, positionnée suivant l'axe de l'électroaimant; l'écoulement est dirigé de telle sorte que le fluide passe d'abord par le polariseur avant d'atteindre la zone d'étude située au centre du solénoïde principal. Ainsi, avant d'être analysés, les spins du liquide en écoulement à la vitesse moyenne $U$ sont soumis au champ magnétique $B_{0}$ sur une distance $h$ de l'ordre de $120 \mathrm{~cm}$. Pour obtenir la relaxation complète des spins le long du champ magnétique $B_{0}$, le temps de polarisation $t_{p}=h / U$ doit être au moins 3-4 fois supérieur au temps de relaxation longitudinale $T_{1}$ des spins. Sinon, seule une fraction $f$ de la polarisation est obtenue ; pour $t_{p} \geqslant T_{1}$, cette fraction $\mathrm{f}$ est bien décrite par la relation suivante:

$$
f=1-\exp \left(-\frac{h}{U T_{1}}\right) \text { pour } t_{p} \geqslant T_{1}
$$

Il convient donc de se limiter à des écoulements dont les vitesses sont inférieures à $U_{\max }=h / T_{1}$. Pour de l'eau pure, $T_{1} \approx 2 \mathrm{~s}$ et $U_{\max }=60 \mathrm{~cm} / \mathrm{s}$. Pour étudier des écoulements à vitesses plus élevées, on peut réduire le temps de relaxation $T_{1}$ en additionnant au fluide des impuretés paramagnétiques (radicaux libres, ions paramagnétiques) ; c'est ainsi qu'en dissolvant $1.45 \mathrm{~g} / \mathrm{l}$ de nitrate de fer dans l'eau, $T_{1}$ est abaissé à $100 \mathrm{~ms}$ et $U_{\max }=12 \mathrm{~m} / \mathrm{s}$.

L'écoulement est étudié dans une canalisation en verre de $50 \mathrm{~mm}$ de diamètre intérieur. Pour effectuer les mesures de RMN dans la zone de haute homogénéité, la canalisation doit impérativement être fabriquée en matériaux diélectriques (verre, céramique, résine) transparents au champ radiofréquence (r.f.). La RMN est étudiée grâce à une bobine r.f., en forme de selle de cheval, ajustée à la canalisation. Cette bobine est utilisée non seulement pour détecter l'induction magnétique mais également pour appliquer des impulsions r.f. destinées à basculer l'aimantation.

Par ailleurs, des impulsions de gradient de champ magnétique suivant l'axe du canal sont délivrées par une bobine de gradient du type quadripolaire [28]. Des gradients de champ variables de 0 à $0,1 \mathrm{Tesla} / \mathrm{m}$ sont appliqués durant les mesures.

Le volume $V$ de fluide étudié dans la zone de haute homogénéité du champ $B_{0}$ correspond à un tronçon de canalisation de longueur $L \approx D$. Dans ce volume, l'homogénéité des gradients de champ magnétique appliqués est estimée à $\pm 1 \%$, et celle du champ radiofréquence, à $\pm 5 \%$. Les déviations maximales apparaissent au voisinage de la paroi du canal.

\section{MÉTHODE RMN UTILISÉE [29]}

Les différentes particules du fluide seront repérées dans un référentiel $(0, x, y, z)$ défini de telle sorte que $O$ soit le centre du volume $V$ et que $(O z)$ soit dirigé suivant l'axe du canal et donc suivant $B_{0}$.

Pour analyser le transport du fluide dans le volume $V$, nous avons appliqué en présence du champ magnétique 
$B_{0}$, une séquence appelée PFGSE (Pulsed Field Gradient Spin-Echo) et présentée figure 2. La séquence commence par une impulsion r.f., notée $(\pi / 2)_{X}$, qui bascule l'aimantation nucléaire du fluide dans le plan perpendiculaire à $B_{0}$. L'aimantation tourne alors dans ce plan à la fréquence de résonance (fréquence de Larmor) $\omega_{0}=\gamma B_{0}$ où le rapport gyromagnétique $\gamma$ dépend des noyaux considérés. Un signal $M_{0}(t)$ est alors détecté. Notons que pour $B_{0}=0,12 \mathrm{Tesla}, \omega_{0}=5 \mathrm{MHz}$ pour les protons et $4,8 \mathrm{MHz}$ pour les noyaux de Fluor

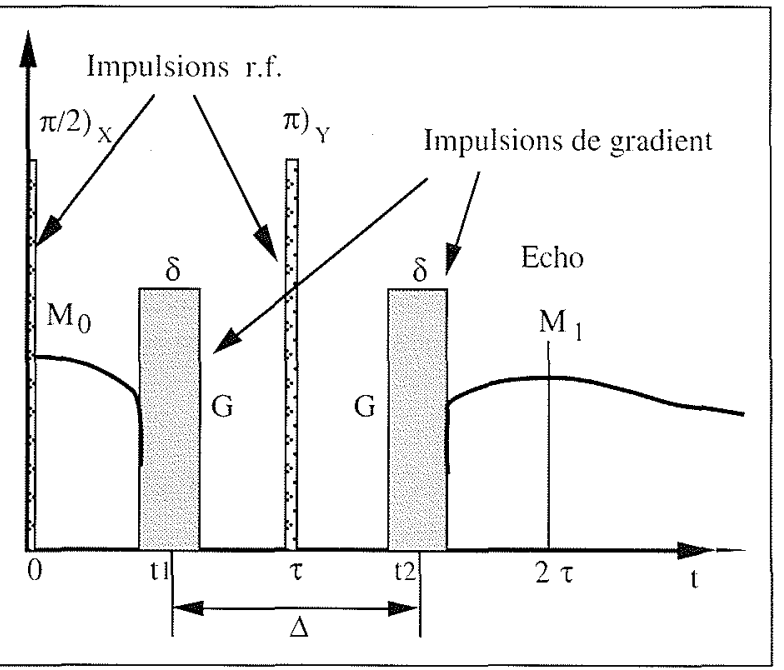

2. Séquence PFGSE utilisée dans les mesures par RMN. En absence des impulsions de gradient de champ, il s'agit d'une séquence classique d'écho de spins où une refocalisation complète de l'aimantation transversale est obtenue au temps de l'écho $(t=2 \tau)$; dans ce cas, aucun effet de l'écoulement n'est observé. En présence des deux impulsions de gradient $(\delta G)$, la phase de l'aimantation transversale est proportionnelle à la vitesse moyenne des spins. Seule une refocalisation partielle de l'aimantation est obtenue à l'instant de l'écho.

Intéressons nous à une famille de spins particulière, ceux des protons par exemple. L'application d'une impulsion de gradient de champ magnétique à l'instant $t_{1}$ va permettre de coder l'espace $V$ : chaque spin va en effet subir dans le plan perpendiculaire $B_{0}$ un déphasage proportionnel à sa position suivant $(O z)$. Ainsi l'aimantation élémentaire d'une particule de fluide $j$ située à l'instant $t_{1}$ à l'ordonnée $z_{j}\left(t_{1}\right)$ subi un déphasage $\phi_{j}\left(t_{1}+\delta\right)$ tel que :

$$
\phi_{j}\left(t_{1}+\delta\right)=\gamma G \int_{t_{1}-\frac{\delta}{2}}^{t_{1}+\frac{\delta}{2}} z(t) \mathrm{d} t
$$

En négligeant les variations de vitesse des noyaux durant $\delta(\approx 1 \mathrm{~ms})$,

$$
\phi_{j}\left(t_{1}+\delta\right)=\gamma G \delta z\left(t_{1}\right)
$$

L'arrivée d'une deuxième impulsion r.f., notée $(\pi)_{Y}$, inverse le signe des déphasages créés précédemment. Le déphasage dans le plan perpendiculaire à $B_{0}$ devient alors :

$$
\phi_{j}\left(t_{1}+\delta\right)=-\gamma G \delta z\left(t_{1}\right)
$$

La seconde impulsion de gradient de champ magnétique, appliqué à l'instant $t_{2}$, induit un déphasage proportionnel à la nouvelle position de la particule $j$. Le déphasage résultant est alors:

$$
\phi_{j}\left(t_{2}+\delta\right)=\gamma G \delta\left[z\left(t_{2}\right)-z\left(t_{1}\right)\right]
$$

Si $\Delta=t_{2}-t_{1}(\approx 2 \mathrm{~ms})$ est plus petit que le temps de corrélation $\tau$, de la vitesse des particules de fluide, on obtient alors à l'instant $2 \tau$ :

$$
\phi_{j}(2 \tau)=\gamma G \delta v(\tau) \Delta
$$

où $v(\tau)$ est la vitesse de la particule de fluide à l'instant

L'aimantation détectée à l'instant $2 \tau$ notée $M_{1}$, est la somme des contributions des différentes particules du fluide contenues dans le volume $V$, de sorte que:

$$
M_{1}(k)=M_{0}<\exp (i k v)>\text { avec } k=\gamma \delta \Delta G
$$

$M_{0}$ est l'aimantation dans le volume $V$ au moment de l'application de la séquence et $<>$ représente une moyenne sur tout le volume $V$.

En introduisant la distribution de probabilité de vitesse $P(v, t)$, où $P(v, t) \mathrm{d} v$ est la probabilité de trouver à l'instant $t$, dans le volume $V$, des particules de fluide avec des vitesses comprises dans l'intervalle $[v, v+d v]$, l'aimantation maximale $M_{1}$, détectée à l'issue d'une séquence appliquée à l'instant $t$, s'écrit :

$M_{1}(t, k)=$

$=M_{0}(t) \int_{-\infty}^{+\infty} \mathrm{d} v P(v, t) \exp (i k v)$ avec $\int_{-\infty}^{+\infty} \mathrm{d} v P(v, t)=1$

$M_{0}(t)$ est l'aimantation présente dans le volume $V$ à l'instant $t$ où la séquence est appliquée.

$\frac{M_{1}(t, k)}{M_{0}(t)}$ apparaît comme la transformée de Fourier de $P(v, t)$ à un instant $t$ donné.

\section{CARACTÉRISATION PAR RMN D'UN ÉCOULEMENT DIPHASIQUE}

Considérons le cas d'un écoulement eau-air dans lequel les mouvements des protons sont analysés par RMN. L'aimantation $M_{0}(t)$ mesurée à l'instant $t$ est proportionnelle au nombre de protons présents dans le volume $V$ à l'instant $t$, et par conséquent, à la fraction volumique $R_{f}(t)$ de l'eau dans le volume $V$. Il s'en suit que:

$$
R_{L}(t)=\frac{M_{0}(t)}{M_{00}}
$$

où $M_{00}$ est l'aimantation mesurée lorsque le canal est plein d'eau $\left(R_{L}=1\right)$.

Ainsi chaque mesure de $M_{0}(t)$ donne accès à la fraction volumique instantanée de l'eau dans le volume $V$. En moyennant les résultats sur une série de mesures, on obtient évidemment la fraction volumique moyenne $\overline{R_{L}(t)}$ :

$$
\overline{R_{L}(t)}=\frac{\overline{M_{0}(t)}}{M_{00}}
$$


Par ailleurs, en développant au premier ordre en $k$ [Martin, DEA Paris VI] la relation (8), on montre que, pour $k$ suffisamment faible, la phase $\Phi_{1}(t)$ de $M_{1}(t)$ est proportionnelle à la vitesse moyenne $v(t)$ de l'eau présente dans $V$ à l'instant $t$ :

$$
\Phi_{1}(t)=k v(t) \text { avec } v(t)=\int_{-\infty}^{+\infty} \mathrm{d} v v P(v, t)
$$

Lorsque $k$ augmente, l'écart $\Phi_{1}(t)-k v(t)$ dépend de l'allure de $P(v, t)$. On montre qu'au troisième ordre en $k$, $\Phi_{1}(t)-k v(t)=-\frac{k^{3} M_{3}(t)}{6}$ où $M_{3}$ est le troisième moment de la distribution $P(v, t)$. Pour le type de distributions de probabilité de vitesse attendu dans les écoulements, on montre par calcul numérique que $\frac{\Phi_{1}(t)-k v(t)}{\Phi_{1}(t)}<1 \%$ tant que $\Phi_{1}<\frac{\pi}{2}$.

La vitesse moyenne instantanée de l'eau dans $V$, $v(t)$, peut donc être déduite facilement de la mesure de la phase $\Phi_{1}(t)$ de $M_{1}(t)$ grâce à la relation linéaire (11). Il convient toutefois de veiller à ce que l'amplitude $G$ du gradient de champ magnétique soit réglée de telle manière que :

$$
G \leqslant \frac{\pi}{2 \gamma \delta \Delta \overline{v(t)}}
$$

Nous allons maintenant examiner une autre possibilité d'exploiter la relation (8). Notons $\overline{M_{0}(t)}$ et $\overline{M_{1}(t, k)}$, les valeurs moyennes dans le temps de $M_{0}(t)$ et $M_{1}(t, k)$, deux valeurs accessibles par RMN. En combinant les équations (8) et (9), on obtient :

$$
\left.\overline{M_{1}(t, k)}=\overline{M_{0}(t)} \int_{-\infty}^{+\infty} \mathrm{d} v \overline{P(v, t)}\right)^{R L} \exp (i k v)
$$

où $\overline{P(v, t)}{ }^{R L}=\frac{\overline{P(v, t) R_{L}(t)}}{R_{L}(t)}$ est la moyenne dans le temps de $P(v, t)$, pondérée par la fraction volumique d'eau présente. La distribution moyenne de probabilité des vitesses $\overline{P(v, t)}{ }^{R L}$ est donc égale à la transformée de Fourier inverse de $\frac{\overline{M_{1}(t, k)}}{M_{0}(t)}$, rapport de deux quantités accessibles par RMN. A partir de $\overline{P(v, t)}$, on peut calculer $v^{R L}$, la moyenne dans. le temps, pondérée par la fraction volumique présente, de la vitesse de l'eau dans le volume $V$ :

$$
\vec{v}^{R L}=\int_{-\infty}^{+\infty} \mathrm{d} v \overline{P(v, t)} \overline{p L}^{R L}
$$

Cette quantité est différente de la vitesse superficielle de l'eau, $J_{L}$, qui est définie par le rapport $\frac{Q_{L}}{A}$, où $Q_{L}$ est le débit volumique en eau, et $A$ la section du canal. Cependant, la vitesse $J_{L}$ peut être reliée aux quantités mesurées par RMN, on peut montrer en effet que :

$$
J_{L}=\overline{R_{L}(t) v(t)} \text { et } J_{L}={\overline{R_{L}(t) v}}^{R L}
$$

En résumé, dans le cas d'un écoulement diphasique eau-air, les grandeurs caractéristiques accessibles à partir des mesures RMN, sont les suivantes:

- les fractions volumiques instantanées et moyennes de l'eau dans le volume $V: R_{L}(t)$ et $\overline{R_{L}(t)}$ [équations (9) et (10)] ;
- la vitesse instantanée de la fraction d'eau dans le volume $V: v(t)$ [équation (11)] ;

- la moyenne dans le temps, pondérée par la fraction volumique présente, de la distribution de probabilité des vitesses de l'eau dans le volume $V: \overline{P(v, t)^{R_{t}}}$ [équation (13)] ;

- la moyenne dans le temps, pondérée par la fraction volumique présente, de la vitesse de l'eau dans le volume $V: v^{R L}$ [équation (14)] :

- la vitesse débitante de l'eau : $J_{L}$ [équation (15)].

Ces résultats permettent une caractérisation du transport de l'eau au sein d'un écoulement diphasique eau-air. Ils peuvent être généralisés immédiatement à des écoulements diphasiques liquide-liquide lorsque les deux liquides sont discernables par RMN. C'est le cas d'un écoulement eauFréon 113 où l'analyse du mouvement des protons perme de caractériser le transport de l'eau, alors que celui du Fréon 113, exempt de proton, est analysable via l'étude par RMN des mouvements des noyaux de Fluor. Les deux phases peuvent donc être analysées indépendamment. Pour les mêmes raisons une analyse séparée des écoulements de chacune des phases liquides peut être réalisée dans un mélange du type eau-Fréon 113-gaz.

\section{PRÉSENTATION DE RÉSULTATS}

\subsection{Boucle diphasique}

La section d'essai est intégrée dans un circuit instrumenté permettant d'entretenir des écoulements stationnaires dans des configurations variées. L'installation a été étudiée et dimensionnée par la Société YLec Consultants (Y. Lecoffre). Le circuit comprend, en suivant le sens de l'écoulement, un mélangeur, la section d'essai, un décanteur rapide permettant la séparation des fluides, deux pompes d'alimentation du mélangeur, munies de dispositifs de réglage et de débitmètres à turbine (précision $\pm 0,8$ litre/mn), une alimentation en air, contrôlée par un débitmètre régulateur massique (précision \pm 2 litres/mn à 1 bar). Deux types de mélangeur sont utilisés. Le mélange eau-Fréon 113 est obtenu grâce à un appareil Optimix qui permet de régler la granulométrie. Le mélange eau-air est obtenu a l'aide d'un dispositif à ogive réglable où l'air est injecté en quatre points dans une zone de décollement.

\subsection{Résultats sur les mélanges eau-Fréon 113}

Les valeurs de fractions volumiques moyennes ont été obtenues en mesurant successivement l'aimantation moyenne des protons et celle des noyaux de fluor sur une série d'écoulements couvrant toute la gamme des fractions volumiques possibles $\left(0<\overline{R_{L \text { eau }}(t)}<1\right)$. Pour tester la cohérence des couples de résultats, chaque couple a été représenté par un point sur la figure 3 dans le plan $\left(\overline{R_{L \text { eau }}(t)}, \overline{R_{L \text { iréon }}(t)}\right)$. La cohérence parfaite des résultats impliquerait que les point représentatifs des mesures soient situés exactement sur la droite $\overline{R_{L \text { Iréon }}(t)}=1-\overline{R_{L \text { eau }}(t)}$. Des écarts de quelques pour cent sont observés sans que l'on puisse affirmer s'ils sont dus à l'imprécision des mesures de RMN ou à de faibles fluctuations dans l'alimentation de la veine d'essai. 


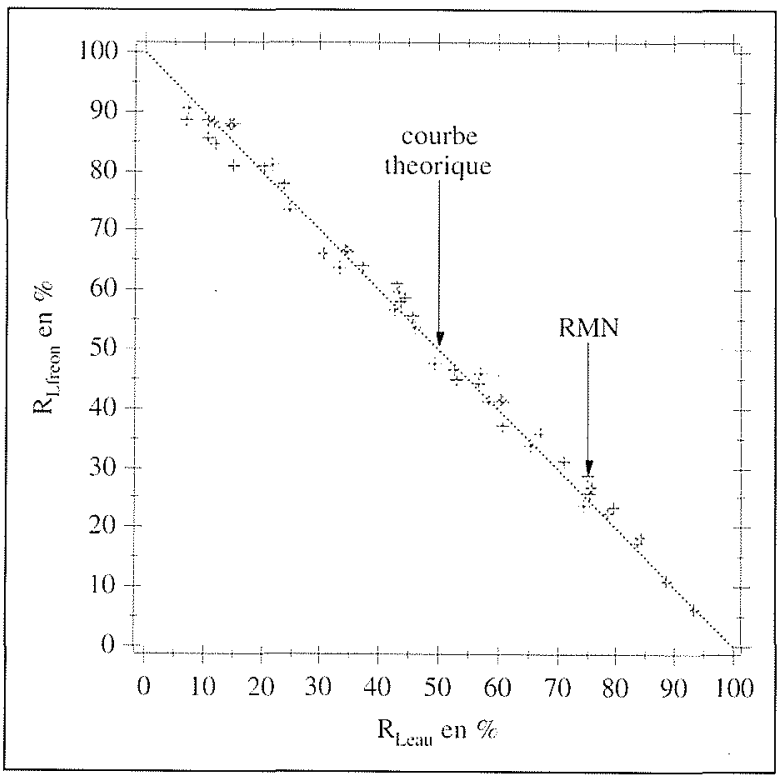

3. Points représentatifs des couples $\left(\overline{R_{L \text { eau }}(t)}\right.$, $\left.\overline{R_{L \text { rréon }}(t)}\right)$ mesurés par RMN. La cohérence des résultats implique que les points représentatifs des mesures soient situés sur la droite $\overline{R_{L \text { fréon }}(t)}=1-\overline{R_{L \text { eau }}(t)}$.

Les figures 4 et 5 présentent les distributions de vitesse obtenues à débit de Fréon constant $\left(J_{L \text { fróon }}=10 \mathrm{~cm} / \mathrm{s}\right)$, pour des débits d'eau variables $\left(J_{\text {Lear }}=18 \mathrm{~cm} / \mathrm{s}\right.$ à $\left.61 \mathrm{~cm} / \mathrm{s}\right)$. Pour des débits d'eau élevés correspondant à $J_{L_{\text {eau }}}=25 \mathrm{~cm} / \mathrm{s}, 40 \mathrm{~cm} / \mathrm{s}$ et $61 \mathrm{~cm} / \mathrm{s}$, le Fréon est dispersé dans l'eau en gouttes d'environ $8 \mathrm{~mm}$ de diamètre; les distributions de probabilité des vitesses, aussi bien pour' l'eau que pour le Fréon, sont des distributions relativement étroites et assez bien symétriques par rapport à la valeur moyenne des vitesses. Ce type de distribution est attendue lorsque les profils des vitesses sont relativement plats pour les deux phases, que la configuration de l'écoulement est stable et que par conséquent les fluctuations des vitesses au sein de chaque phase reste de faible amplitude. On constate toutefois que les largeurs des distributions des vitesses mesurées dans l'eau sont grosso modo deux fois supérieures à celles des distributions mesurées dans le Fréon ; ce qui signifierait que les fluctuations de vitesse dans la phase dispersante sont beaucoup plus importantes que dans la phase dispersée.

Lorsque les débits d'eau sont plus faibles $\left(J_{\text {Leau }}=15 \mathrm{~cm} / \mathrm{s}, 18 \mathrm{~cm} / \mathrm{s}\right.$ et $\left.21 \mathrm{~cm} / \mathrm{s}\right)$, les largeurs des distributions des vitesses deviennent très importantes et égales dans les deux phases. Cet effet est cohérent avec l'apparition d'instabilités de configuration dans l'écoulement, instabilités qui s'accompagnent de fuctuations très importantes des vitesses dans les deux phases.

Comme cela a été signalé plus haut, l'étude RMN permet de déterminer dans un écoulement diphasique les vitesses superficielles des deux phases, $J_{L \text { fréon }}$ et $J_{L \text { eau }}$. Par ailleurs, ces mêmes grandeurs peuvent être immédiatement déduites des débits mesurés par les débitmètres à turbine placés sur les tubes d'alimentation du mélangeur. Les figures 6 et 7 permettent d'apprécier la cohérence des valeurs des vitesses superficielles obtenues par les deux méthodes.

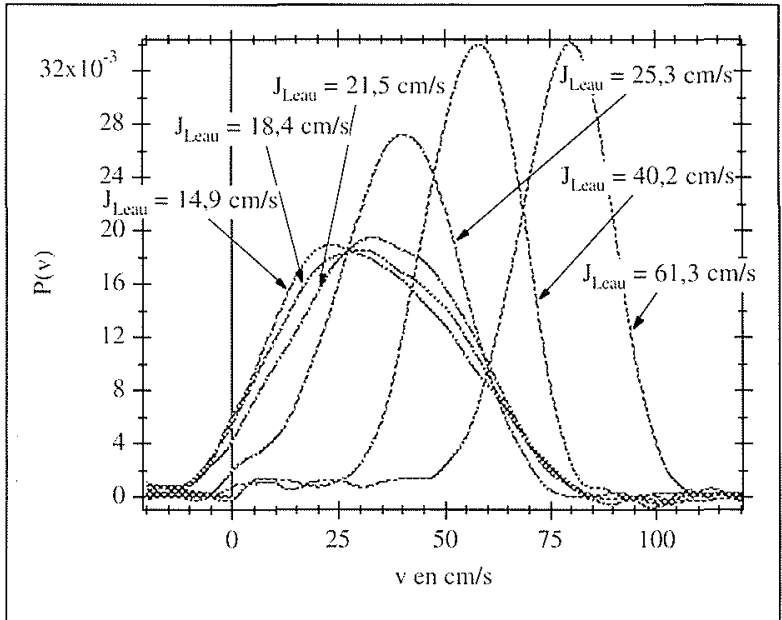

4. Distributions de probabilité des vitesses dans I'eau, $\overline{P(v, t}^{R L}$ eau, obtenues à débit de Fréon constant $\left(J_{L \text { fréon }}=10 \mathrm{~cm} / \mathrm{s}\right)$ pour des débits d'eau variables $\left(J_{L \text { eau }}=18 \mathrm{~cm} / \mathrm{s}\right.$ à $\left.61 \mathrm{~cm} / \mathrm{s}\right)$.

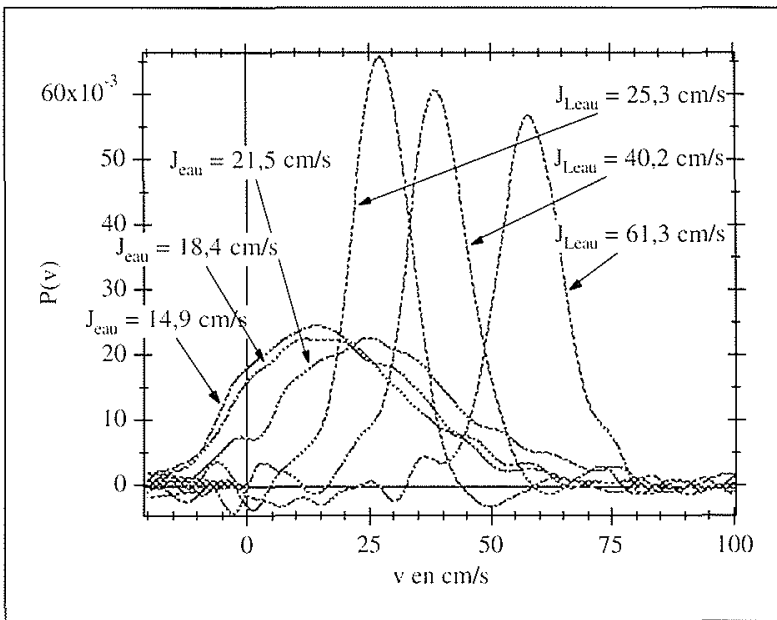

5. Distributions de probabilité des vitesses dans le Fréon,

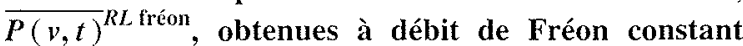
$\left(J_{L \text { fréon }}=10 \mathrm{~cm} / \mathrm{s}\right)$ pour des débits d'eau variables $\left(J_{L \text { eaut }}=18 \mathrm{~cm} / \mathrm{s}\right.$ à $\left.61 \mathrm{~cm} / \mathrm{s}\right)$.

\subsection{Résultats sur les mélanges eau-air}

Les distributions de probabilité des vitesses de l'eau dans les écoulements diphasiques eau-air sont assez différentes de celles observées dans les écoulements diphasiques liquide-liquide. Même pour de faibles taux de vide (écoulements à bulles), les distributions de probabilités des vitesses sont fortement élargies par rapport a celle qui correspond à un écoulement d'eau seule. Cet élargissement est attribué à l'apparition de fluctuations importantes des vitesses engendrées par les bulles. Au-delà d'un taux de vide de $20 \%$, la configuration de l'écoulement devient instable et les fluctuations de vitesse atteignent plus de $100 \%$ de la vitesse moyenne de l'eau. Ainsi pour $J_{V}=29,5 \mathrm{~cm} / \mathrm{s}$ et $J_{G}=32 \mathrm{~cm} / \mathrm{s}$ (correspondant à un taux de vide, $R_{G}$, de $42 \%$ ), la distribution des vitesses s'étale de $-0,5 \mathrm{~m} / \mathrm{s}$ à $1,5 \mathrm{~m} / \mathrm{s}$ 


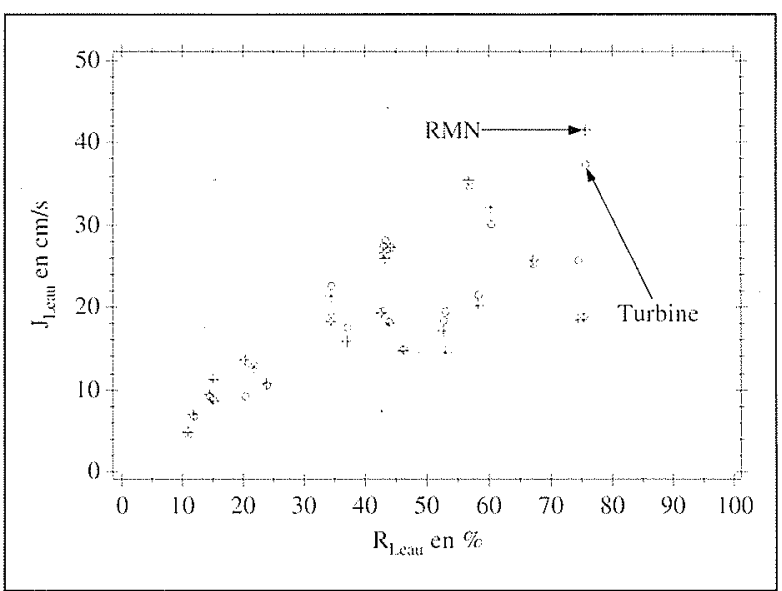

6. Sur le même graphe sont représentés, pour différentes fractions volumiques d'eau, les vitesses superficielles de l'eau mesurées par RMN + et par débitmétrie classique $o$.

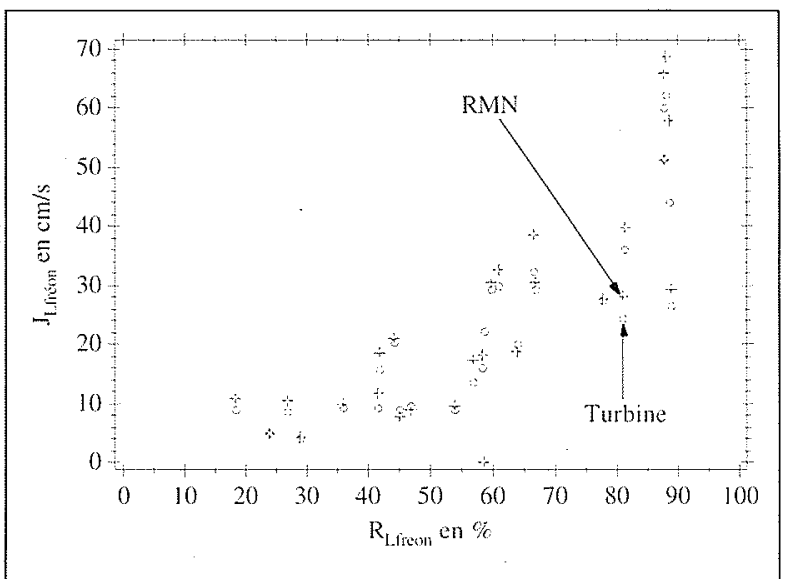

7. Sur le même graphe sont représentés, pour différentes fractions volumiques de Fréon, les vitesses superficielles du Fréon mesurées par RMN + et par débitmétrie classique $o$.

La figure 9 permet de comparer les valeurs des vitesses superficielles déduites des mesures de RMN avec celles obtenues par débitmétrie classique. On constate qu'il y a bon accord sur une large gamme de fraction volumique.

\section{LIMITES ACTUELLES DE LA MÉ- THODE ET PERSPECTIVES}

Dans sa configuration actuelle, Spinflow n'est applicable qu'à des écoulements à vitesse modérée, n'excédant pas 1 ou $2 \mathrm{~m} / \mathrm{s}$. In ne s'agit pas d'une limitation théorique, mais essentiellement technique, liée à un problème de commutation du gradient de champ magnétique utilisé dans la séquence. Cette difficulté, bien connue en imagerie RMN, limite les cadences d'acquisition des images. En fait il ne s'agit pas d'un simple problème de commutation de courant d'alimentation de la bobine de gradient. La bobine utilisée, du type quadripolaire, présente une faible induc-

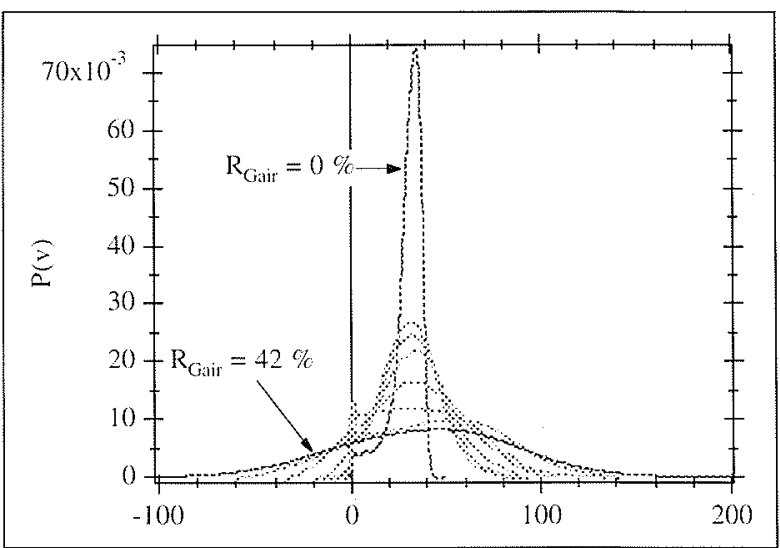

8. Distributions de probabilité des vitesses dans l'eau, $\overline{P(v, t)^{R L} \text { eau }}$, obtenues à débit d'eau constant dans un mélange eau-air pour des taux de vide variant de $0 \%$ à $42 \%\left(J_{L \text { eau }}=29,5 \mathrm{~cm} / \mathrm{s} ; J_{G}=0\right.$ à $\left.32 \mathrm{~cm} / \mathrm{s}\right)$.

tion et la commutation du courant peut être réalisée en moins de $100 \mu \mathrm{s}$. Le problème est ailleurs ; il provient du couplage magnétique inévitable entre la bobine et les éléments extérieurs. L'installation d'un dispositif électronique de correction a permis de compenser en partie ces effets en réduisant le temps de commutation à environ $500 \mu \mathrm{s}$. Dans ces conditions, le temps minimum d'application de la séquence PFGSE est de l'ordre de $2 \tau=3,6 \mathrm{~ms}$ (avec $\Delta=1,8 \mathrm{~ms}$ et $\delta=600 \mu \mathrm{s}$ ). Durant ce temps, les déplacements des particules de fluide doivent être négligeables par rapport à la dimension du volume de mesure $(L=D)$; cela implique :

$$
v \leqslant 0,1 \frac{L}{2 \tau} \quad \text { (dans le cas présent } v \leqslant 1,4 \mathrm{~m} / \mathrm{s} \text { ) }
$$

Au-delà de cette limite, on peut envisager une correction prenant en compte les pertes du fluide marqué dans le volume de mesure durant la séquence. Si le temps de corrélation de la vitesse des particules de fluide, $\tau_{p}$, est négligeable devant la durée $2 \tau$ de la séquence, on montre facilement que :

$$
P(v, t) \text { mesuré par RMN } \infty P(v, t)\left(1-\frac{2 \tau(v)}{L}\right)
$$

Cette correction est pratiquement envisageable jusqu'à des vitesses maximales de $0,5 \frac{L}{2 \tau}$, soit $7 \mathrm{~m} / \mathrm{s}$.

Le système. Spinflow, tel qu'il se présente aujourd'hui est un système performant, très facile à maîtriser, et ne nécessitant de connaître que quelques rudiments de RMN. Il s'agit d'un équipement qui a priori a sa place au sein de nombreuses équipes de mécaniciens des fluides spécialisés dans les écoulements multiphasiques.

La méthode d'analyse des écoulements utilisée dans le dispositif est compatible avec les techniques d'imagerie RMN, de sorte qu'au-delà des performances actuelles on peut envisager à court terme d'obtenir dans une conduite les profils des fractions volumiques et des vitesses de chaque phase [30]. Une extension de ce type présente dès aujourd'hui un grand intérêt puisqu'un effort en modélisation d'écoulements diphasiques porte depuis peu sur la mise au point de modèles numériques multidimensionnels. 


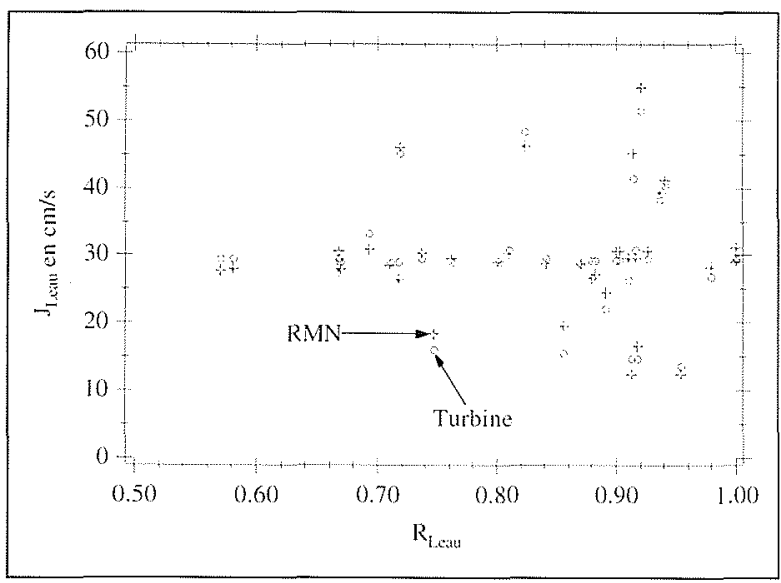

9. Sur le même graphe sont représentés pour différentes fractions volumiques d'eau les vitesses superficielles de l'eau mesurées par RMN + et par débitmétrie classique 0 .

\section{Références}

[I] STEJSKal E.O. (1965). - Use of spin-echos in a pulsed magnetic field gradient to study anisotropic, restricted diffusion and flow, J. Chem. Plyys, 43, 3597-3603.

[2] Stejskal E.O. \& Tanner J.E. (1965). - Spin diffusion measurements: spin echoes in the presence of a timedependent field gradient, J. Chem. Phys., 42, 288-292.

[3] Packer K.J. (1969): - The study of slow coherent molecular motion by pulse nuclear magnetic resonance, Mol. Phys., 17. 355-368.

[4] Kruger H., Spiesecke H., Van Steenwinkel R., Noack F. (1977), - Nuclear magnetic relaxation and selfdiffusion in a series of $p$-alkanoyl-benzylidene, $p$ 'aminoazobenzenes, Mol. Cryst. Liq. Cryst., 40, 103-116.

[5] Callaghan P.T. (1984). - Pulsed field gradient nuelear magnetic resonance of liquid state molecular organization, Aust. J. Phys., 37, 359-387.

[6] Grover Th. \& Singer J.R. (1971). - NMR spin echo flow measurements, J. Appl. Phys., 42, 938-940.

17] Deville G. \& Landesman A. (1971). - Expériences d'échos de spin dans un liquide en écoulement, Journal de Physique, 32, 67-72.

[8] Hayward R.J., Pecker K.J. \& Tomlinson D.J. (1972). Pulsed field-gradient spin-echo NMR studies of flow in fluids, Mol. Phys., 23, 1083-1102.

[9] GaRroway A.N. (1974). - Velocity measuiements in flowing by NMR, J. Phys, D7, L159-L163.

[10] DE GENNES P.G. (1969). - Theory of spin-echoes in a turbulent fluid, Phys. Lett., 29A. 200-21.

[11] CARR H.Y. \& PURCELL E.M. (1954). - Effects of diffusion on free precession in nuclear magnetic resonance experiments. Phys. Rev. 94, 630-638.

[12] FUKUdA K. \& HiRai A. (1979). - A pulsed NMR study on the flow of thuid, J. Phys. Soc. Jap., 47, 1999-2000.

[13] KaWAbE Y., FukUda K. \& HiRAI A. (1983). - A note on the signal intensity of the second spin-echo in Carr-PurcellMeiboom-Gill sequence of turbulent flows. J. Phys. Soc. Jap., 52, 2308-2311.
[14] Bogdanov P.I. \& Zykov P.G. (1984). - Influence of convective, hydrodynamic and diffusive transport on spin-echo signals, Sov: Phys. Tech. Phys., 29, 494-495.

[15] KOSE K... SATOH K., INOUYE T. \& YASUOKA H. (1985). NMR flow imaging, J. Phys. Soc. Jap., 54, $81-92$

116] Singer J.R. (1978). - NMR diffusion and flow measurements and an introduction to spin phase graphing, J. Phys. E., 11, 281-291.

[17] Battocletth J.H., Halbach R.E., Salles-Cuniba S.X. \& Sances A. (1981). - The NMR blood fiow-meter-theory and history, Med. Phys., 8, 435-443.

[18] Hemminga M.A. (1984). - Measurement of flow characteristics using nuclear magnetic resonance in biomedical magnetic resonance, James T.L. \& Margulis A.R., Eds, Radiology Research and Education Foundation, San Francisco, 157-184.

[19] STEPISNIK J. (1985). - Measuring and imaging of flow by NMR, Prog. Nucl. Magn. Resonance imaging to visualization of flow in porous media, Spectrosc.. 17, 187-209.

[20] Caprihan A. \& Fukushima E. (1990). - Flow measurements by NMR, Physics Reports, 198, No 4, 195-235.

[21] Gaigalas A.K.., VAN ORden A.C.L., Robertson B., MARECI T.H. \& LEWIS L.A. (1984). - Application of magnetic resonance imaging to visualization of flow in porous media, Nucl. Technol, 84, 113

[22] Edelstein W.A., Vinegar H.J., TUtuJAN P.N., Rogmer P.B. \& Mueller O.M. (1988). - NMR imaging of core analysis, Proc. 63th Annu. Tech. Conf. and Exhib., Soc. Petrolum Engineers, SPE 18272, 101-112.

[23] Majors P.D., Smith J.L., Kovarik F.S. \& Fukushima E. (1990). - NMR spectroscopic imaging of oil displacement in folomite, J. Magn. Resonance, 89, 470-478.

[24] LYNCH G.F. \& SEGEL S.L. (1977). - Direct measurement of the void fraction of a two phase fluid by nuclear magnetic resonance, Int. J. Heat Mass. Transfer; 20, 7-14.

[25] Abouel wafa M.S.A. \& Kendall E.J.M. (1979). - Optimization of continuous wave nuclear magnetic resonance to determine in situ volume fractions and individual flow rates in two component mixtures, Rev. Sci. Instrum., 50, 1545-1949.

[26] KRÜger G.J., HaUpt J. \& Weiss R. (1984). - A nuclear magnetic resonance method for the investigation of twophase flow, Measuring Techniques in Gas-Liquid Two Phase Flows, Delhaye J.M. \& Cognet G., Eds, Springer-Verlag, $435-454$

27] Majors P.D., Givler E.C. \& FuKLISHIMA E. (1989). Velocity and concentration measurements in multiphase flows by NMR, J. Magn. Res. 85, 235-243.

[28] Webster D.S. \& MARsden K.H.. (1974). - Improved apparatus for NMR measurements of self diffusion coefficients using pulsed field gradient. Rev. Sci. Instrum., 45, $1232-1234$

[29] LeBlond J., Benkedda Y., Javelot S. \& OGer L. (1994), - Two-phase flows by Pulsed Field Gradient Spin-Echo NMR, Meas. Sci \& Techn. (à paraître).

[30] Leblond J. \& Stepowsky D. (1994). - Some nonintrusive methods for diagnosis in two-phase flows, Multiphase Science and Technology (à paraître). 